\title{
Detection of DNA sequences using an alternating electric field in a nanopore capacitor
}

\author{
Grigori Sigalov $\dagger$, Jeffrey Comer ${ }^{\dagger,}{ }^{*}$, Gregory Timp ${ }^{\dagger}$, and Aleksei Aksimentiev ${ }^{\dagger},{ }^{*}, 1$ \\ $\dagger$ Beckman Institute for Advanced Science and Technology University of Illinois at Urbana-Champaign, 405 \\ North Mathews, Urbana, IL 61801, USA \\ *Department of Physics University of Illinois at Urbana-Champaign, 405 North Mathews, Urbana, IL 61801, \\ USA
}

\section{Abstract}

Molecular dynamics simulations revealed that back-and-forth motion of DNA strands through a 1$\mathrm{nm}$-diameter pore exhibits sequence-specific hysteresis that arises from the reorientation of the DNA bases in the nanopore constriction. Such hysteresis of the DNA motion results in detectable changes of the electrostatic potential at the electrodes of the nanopore capacitor and in a sequence-specific drift of the DNA strand under an oscillating transmembrane bias. A strategy for sequencing DNA using electric-field pulses is suggested.

High-throughput technology for sequencing DNA has already provided invaluable information about the organization of the human genome ${ }^{1}$ and the common variations of the genome sequence among groups of individuals. ${ }^{2}$ To date, however, the high cost of whole-genome sequencing limits widespread use of this method in basic research and personal medicine. Among the plethora of sequencing methods under development ${ }^{3,4}$ that promise dramatic reduction of genome sequencing costs, the so-called nanopore methods ${ }^{5,6}$ are among the most revolutionary. The main advantage of the nanopore method is that the sequence of nucleotides can be detected, in principle, directly from the DNA strand via electric recording, ${ }^{7-18}$ requiring minimal reagents and having no limits on the length of the DNA fragment that can be read in one measurement.

In this manuscript we investigate the feasibility of sequencing DNA using an alternating electric field in a nanopore capacitor. Through molecular dynamics (MD) simulations we demonstrate that back-and-forth motion of DNA in a 1-nm-diameter pore has a sequencespecific hysteresis that results in a detectable change of the electrostatic potential at the electrodes of the nanopore capacitor and in a sequence-specific drift of the DNA strand through the pore under an oscillating bias. Based on these observations, we propose a method for detecting DNA sequences by modulating the pattern of the applied alternating potential. We consider a single nanopore in a multilayered silicon membrane submerged in an electrolyte solution, Fig. 1. The capacitor membrane consists of two conducting layers (doped silicon) separated by a layer of insulator (silicon dioxide). An external electric bias $V_{\mathrm{ex}}$ is applied across the membrane to drive a single DNA strand back and forth through the pore, while the electric potentials induced by the DNA motion are independently recorded at the top and bottom layers (electrodes) of the capacitor membrane, $V_{\text {top }}$ and $V_{\text {bot }}$, respectively. Nanometer-diameter pores in such membranes have already been manufactured, $19-21$ and the voltage signals resulting from the translocation of DNA strands through such pores have been recorded. ${ }^{22}$ In particular,

\footnotetext{
${ }^{1}$ Corresponding author. E-mail: aksiment@uiuc.edu
} 
measurements have recently been reported using a single DNA molecule trapped in a nanopore of a $3.4 \mathrm{~nm} \times 4.2 \mathrm{~nm}$ cross section in a MOS capacitor with an area of $10 \mu \mathrm{m} \times 10 \mu \mathrm{m}$ and a gate dielectric nominally $2 \mathrm{~nm}$ thick. ${ }^{23}$ The measured frequency response of this device extends beyond $2 \mathrm{MHz}$ (to an upper corner frequency $3.6 \mathrm{MHz}$ according to a lumped element model) and is determined predominately by the electrolytic resistance, which is about $50-100 \mathrm{k} \Omega$ for $\mathrm{KCl}$ concentration in the $100 \mathrm{mM}$ to $1 \mathrm{M}$ range, and a parasitic capacitance associated with the membrane $(<10 \mathrm{pF})$. The improvement in the bandwidth over patch clamping and prior measurements on hemolysin ${ }^{7-10}$ and synthetic pores ${ }^{11-16}$ can be attributed to miniaturization and the concomitant reduction of parasitics. Based on measurements like these, a twodimensional scaling rule, and the corresponding equivalent circuit, one can expect to recover an improvement in the upper corner frequency from 3.6 to $47 \mathrm{MHz}$ using a $2 \mu \mathrm{m} \times 2 \mu \mathrm{m}$ MOS capacitor and to $2.8 \mathrm{GHz}$ for an area of $0.2 \mu \mathrm{m} \times 0.2 \mu \mathrm{m}$. All of these specifications are within the grasp of current technology.

An all-atom model of a nanopore, DNA, and electrolyte was constructed using protocols described previously. ${ }^{24}$ A 10.3 -nm-thick $\mathrm{Si}_{3} \mathrm{~N}_{4}$ membrane was build by replicating a unit cell of a $\beta-\mathrm{Si}_{3} \mathrm{~N}_{4}$ crystal. Removing atoms from the membrane produced an hourglass pore, whose shape closely resembles that of the pores sputtered by a highly-focused electron beam. 25,26 In the middle of the membrane, the pore's shape is a cylinder of a 1-nm diameter and a 1-nm height. The pore gradually opens to both sides of the membrane at a $30^{\circ}$ angle. The pore is symmetric relative to the vertical axis $(x=y=0)$ and the membrane's midplane $(z=0)$. Note that we use the same materials $\left(\mathrm{Si}_{3} \mathrm{~N}_{4}\right)$ to model both the conducting and insulating layers, as in this study we do not explicitly consider the electronic degrees of freedom of the conductor and use an approximate method to compute the electrostatic potentials induced by DNA.

Four systems were produced by merging the nanopore with four DNA strands each made entirely from 25 adenine, cytosine, guanine, or thymine DNA nucleotides. The DNA strands were oriented along the pore axis and threaded halfway through the pore; some DNA bases had to be tilted to fit the constriction (see Fig. 1). Each system was then solvated in a preequilibrated volume of TIP3 water. $\mathrm{K}^{+}$and $\mathrm{Cl}^{-}$ions were added to obtain a $1 \mathrm{M} \mathrm{KCl}$ concentration and render the overall system neutral. The total number of atoms in each system was $\sim 53,500$. Following the assembly, each system underwent 1,000 steps of minimization using a conjugate gradient method. The temperature of each system was increased from 0 to $295 \mathrm{~K}$ in $5 \mathrm{ps}$, followed by a $2 \mathrm{~ns}$ equilibration in the $\mathrm{NpT}$ ensemble (constant number of particles, pressure and temperature) at 1 bar. The production runs were carried out in the $N V$ $T$ ensemble (constant number of atoms, volume and temperature) at $295 \mathrm{~K}$ maintained using a Langevin thermostat ${ }^{27}$ with a damping constant of $10 \mathrm{ps}^{-1}$ applied to all heavy atoms. The atoms of the $\mathrm{Si}_{3} \mathrm{~N}_{4}$ membrane were restrained to their initial positions in the crystal lattice by harmonic forces using spring constants of $10 \mathrm{kcal} / \mathrm{mol} \AA^{2}$ for surface atoms and $1 \mathrm{kcal} / \mathrm{mol}$ $\AA^{2}$ for bulk atoms. All MD simulation were carried out using the program NAMD, 28 -fs time step, AMBER $^{29}$ force field describing DNA and electrolyte, and a custom force field describing $\mathrm{Si}_{3} \mathrm{~N}_{4} .{ }^{30}$ Other simulation protocols are described elsewhere. ${ }^{24}$

A test simulation carried out by applying a sine-wave external bias revealed a strong dependance of the DNA velocity of the translocation direction, $, 31,32$ leading to DNA escape from the pore after just two oscillations of the external bias. To enable observation of multiple translocation cycles in one simulation and simplify analysis of the MD trajectories, a squarewave external potential was applied in all subsequent simulations (see Fig. 2h). The duration of the constant-bias fragments of the square-wave potential was adjusted to produce displacement of DNA with the amplitude of $25 \AA$ (a smaller amplitude was allowed in the first two cycles). Since the displacement amplitude was nearly constant while the DNA's mobility fluctuated, the duration of the individual constant-bias fragments fluctuated respectively. Such adjustment of the applied bias is not required for the sequencing method that is proposed below. 
A relatively large amplitude of the applied bias, $20 \mathrm{~V}$, was chosen to ensure that $15-20$ cycles of DNA oscillation are observed within $\sim 50 \mathrm{~ns}$.

Fig. 2a-g illustrate the changes in the DNA conformation accompanying DNA's back-andforth motion during one translocation cycle. Note that, in the pore constriction, the DNA bases always tilt in the direction opposite to the direction of DNA motion. Fig. $2 \mathrm{~h}$ illustrates the applied transmembrane potential, whereas Fig. 2i plots the $z$-coordinate of a DNA nucleotide passing through the pore constriction. Each time a nucleotide re-enters the nanopore constriction, its base slants back (cf. Fig. 2b,e). Such reorientation of the base leads to a change of the vertical component (projected on the pore axis) of the nucleotide's dipole moment, as shown in Fig. 2j. The dipole moment of the backbone changes according to the translocation direction as well, although it accounts for only a minor part of the total dipole moment variation. An animation illustrating the back-and-forth motion of a DNA strand through this nanopore is available in the supporting information.

Although each DNA nucleotide carries the same overall charge (-e), one can, in principle, distinguish the type of a DNA nucleotide by measuring its dipole moment. Our simulations have demonstrated that the projection of a nucleotide's dipole moment on the pore axis, $D_{z}$, periodically changes during the DNA oscillation in a nanopore. Such repetitive variations of the dipole moment may, conceivably, alter the nanopore capacitance, and hence be detectable. In Fig. 3 we characterize the change of the dipole moment projection for each type of DNA homopolymer oscillating in the nanopore. For each system, we compute $D_{z}$ of each nucleotide from the 54-ns MD trajectory sampled every picosecond. Next, we select fragments from the trajectories of individual nucleotides conforming to the following criteria: at the beginning of the fragment the nucleotide is located at the center of the membrane $(z \approx 0)$; within one turnover of the driving potential that nucleotide leaves the pore's constriction $(z= \pm 5 \AA)$, re-enters the pore, and returns to the membrane's center. We call such a trajectory fragment a partial cycle. Partial cycles obtained by this method are grouped together using as a parameter the maximum displacement of the nucleotide from the center of the membrane $z^{*}$. Such a procedure allows for modeling oscillations of the nucleotides at any amplitude smaller than that actually observed in the MD trajectories. Thus, for a given turning point $z^{*}$, the average $D_{z}(z)$ computed for each translocation direction describes the variation of the nucleotide's $D_{z}$ during an oscillatory motion with the amplitude of $\left|z^{*}\right|$. Fig. 3 shows such statistical dependences obtained for the DNA oscillation with the amplitudes of $15 \pm 5,25 \pm 5$, and $35 \pm 5 \AA$. The dipole moment exhibits a hysteresis for every sequence considered. Within the pore's constriction, the amplitude of the hysteresis does not depend on the amplitude of the DNA oscillation, but depends on the DNA sequence. The hysteresis of the cytosine homopolymer differs the most from the other homopolymers considered. At amplitudes $z^{*}<10 \AA$, the rotation of bases becomes sterically suppressed, and the hysteresis considerably decreases (data not shown). Therefore, in order to observe the hysteresis of the dipole moment, the DNA must oscillate with a minimum amplitude of $\sim 10 \AA$ (i.e., from $-10 \AA$ to $+10 \AA$ ), which is approximately equal to the span of the three nucleotides stretched through the constriction of the pore.

The transformation of the DNA conformation observed during the DNA motion produces timedependent changes of the electrostatic potential at the electrodes of the capacitor. For simplicity, we compute in this study the induced potential by approximating each electrode by twelve point "probes" uniformly distributed around the pore's axis at the electrode's level $z_{\text {elec }}$, each probe being buried inside the membrane $5 \AA$ away from the nanopore's surface. The potential at each electrode is calculated as $\phi\left(z_{\text {elec }}\right)=\frac{1}{12 \epsilon} \Sigma_{i} \Sigma_{j} q_{j} \exp \left(-\kappa\left|\mathbf{r}_{j}-\mathbf{R}_{i}\right|\right) /\left|\mathbf{r}_{j}-\mathbf{R}_{i}\right|$,

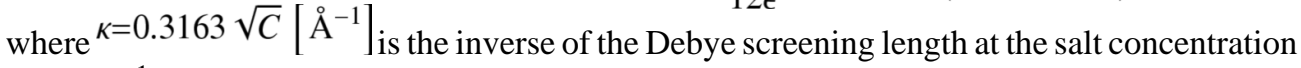
$C\left[\mathrm{~mol} \mathrm{l}^{-1}\right], \epsilon=80$ is the dielectric constant of the solvent, $\mathbf{R}_{i}=\left(x_{i}, y_{i}, z_{i}\right)$ is the location of the 
point probe, $\mathbf{r}_{j}$ and $q_{j}$ are the position and the charge of an atom, and the sums run over all point probes and all DNA atoms.

The time dependence of the potentials induced at the two electrodes located at the edge of the nanopore constriction $\left(z_{\text {elec }}= \pm 5 \AA\right)$ are shown in Fig. $4 \mathrm{a}$. The potentials are observed to change within the range of several millivolts, in agreement with preliminary experimental data. ${ }^{22} \mathrm{~A}$ strong dependence of the induced potential on the translocation direction is observed at the top electrode, but little or no dependence is observed at the bottom electrode. Note that while the nanopore membrane is symmetric relative to the $z=0$ midplane, the DNA is not, as it is oriented with its $3^{\prime}$ end up in all our systems. Thus, the top electrode is facing the DNA's 3 ' end, whereas the bottom electrode is facing the DNA's $5^{\prime}$ end. To characterize the induced potentials in statistical terms, the probability histograms were computed separately for the top, Fig. 4b, and bottom, Fig. 4c, electrodes and for the $5^{\prime}$ and $3^{\prime}$ translocation directions, the latter being controlled by the polarity of the applied bias. The data for all types of the DNA homopolymer are presented in Fig. S1 in the supporting information. The four average values (also most probable within the sampling accuracy) of the induced potentials uniquely characterize the type of the homopolymer confined in a nanopore. To demonstrate that, in Fig. 4d, we plot the difference of the induced potential at the top and bottom electrodes upon switching the polarity of the driving bias. To verify these results, we performed the same analysis on MD trajectories obtained by driving $(\mathrm{dG})_{70}$ and $(\mathrm{dT})_{70}$ strands through the same nanopore at a constant bias of +20 or $-20 \mathrm{~V}$. In the constant bias simulations, the induced potentials were observed to have similar distributions to those obtained under the alternating field conditions (Fig. S2).

Analogous simulations using a DNA block copolymer suggest a possibility of resolving blocks of four identical nucleotides (Fig. S3).

As shown by direct calculation (Fig. S4), the major contribution to the induced potential originates from the DNA backbone atoms. Among those, the highly charged phosphate group $\left(\mathrm{PO}_{4}\right)$ accounts for the most of the total potential, in agreement with previous calculations. ${ }^{33}$ During the translocation towards the $5^{\prime}$ end of the strand, the phosphate group of a nucleotide is pushed against the pore wall by the base of the preceding nucleotide. In the case of the $3^{\prime}$ end-bound translocation, the nucleotide's base is tilted towards the nucleotide's phosphate. Such a conformation makes the nucleotide's cross section in the $x y$ plane slightly smaller when compared to that typically observed in the $5^{\prime}$ end-bound translocation. Hence, for the $3^{\prime}$ endbound translocation, the phosphate group is found, on the average, $\sim 1 \AA$ farther from the pore's wall (and the electrodes) than in the case of the $3^{\prime}$ end-bound translocation. Consequently, the absolute values of the induced potentials are systematically smaller for the $3^{\prime}$ end-bound translocations.

Although most of the induced potential arises from the charge of the DNA backbone, the chemical identity of the bases affects the induced potential in the following two ways. First, the dipole moment of the base directly contributes to the total potential. Second, through steric hindrances the base modulates the potential induced by the backbone, as the backbone's position relative to the electrodes depends on the size of the base and the base's ability to tilt in the nanopore's constriction. Hence, the ensuing differences in the potential are base typespecific and can be employed for DNA sequencing, upon careful design and calibration of the device.

Our MD simulations suggest that in order to discriminate the sequence of DNA by measuring the electrostatic potential in a nanopore capacitor, the experimental device should be capable of distinguishing a submicrovolt difference in the potential within the time interval dictated by the rate of DNA transport between the plates of the capacitor. In our simulations, we employed a high frequency bias to produce statistically significant number of DNA nucleotide permeation events and thereby average the induced potentials over many DNA conformations. We found 
that in a 1-nm-diameter pore, DNA conformational disorder is greatly suppressed, because the DNA strand is guaranteed to have a single-file conformation. Hence, the conformational disorder is limited to the rotation of the bases within the plane of the membrane, which is averaged out in the measurement of the induced potential because of the cylindrical symmetry of the electrodes. We anticipate that the experimental measurements will be conducted at a much lower frequency and amplitude of the alternating bias, which would enable averaging over the conformational disorder within one permeation event.

It is important to stress that our calculations did not take into account other noise sources, which could arise from the stochastic motion of ions and water, telegraph noise in the semiconductor, parasitic capacitance, etc. Taking into account parasitics, the signal associated with the charge of a single phosphate is predicted to be $\sim 50 \mu \mathrm{V}$ and that from a DNA base at $\sim 10 \mu \mathrm{V}{ }^{23}$ As argued below, $10-50 \mu \mathrm{V}$ could be a significant signal. For example, using synchronous, lockin detection to study DNA trapped in a nanopore in an MOS capacitor signals $<100 \mathrm{nV}$ can easily be detected. ${ }^{23}$ To see how this works, suppose that we trap a long strand of DNA in a nanopore and use an ac-sinusoidal electric field at a frequency of $1 \mathrm{MHz}$ along the pore axis to modulate the position of the molecule between the electrodes, resulting in a sine-wave signal on the ideal electrodes $>10 \mu \mathrm{V}$. (We will assume that all the contributions to the measured rmsvoltage are statistically uncorrelated, so that the noise adds in quadrature.) Using an amplifier with input noise of $<4 \mathrm{nV} \mathrm{Hz}^{-1 / 2}$ at a bandwidth of $100 \mathrm{MHz}$ and a gain of 1000 , the signal to-be-measured will be about $10 \mathrm{mV} \approx(1000 \times 10 \mu \mathrm{V})$ along with $40 \mathrm{mV} \approx 4 \mathrm{nV} \mathrm{Hz}^{-1 / 2} \times 1000 \times$ $(100 \mathrm{MHz})^{1 / 2}$ of broadband RMS noise, making it difficult to discriminate the signal from the noise. On the other hand, if the same signal is fed from the pre-amp into a lock-in amplifier that can detect a frequency of $1 \mathrm{MHz}$ with a bandwidth of $1 \mathrm{kHz}$, and the output time constant is $1 \mathrm{~ms}$, then the RMS noise accompanying the signal would be only $130 \mu \mathrm{V} \approx 4 \mathrm{nV} \mathrm{Hz}^{-1 / 2} \times$ $1000 \times(1000 \mathrm{~Hz})^{1 / 2}$ instead of $40 \mathrm{mV}$, indicating that it should be possible to extract a $10 \mu \mathrm{V}$ input signal.

Before discussing the possibility of distinguishing individual nucleotides, we examine the character of DNA motion in the pore. As shown in Fig. 2i, the motion of DNA nucleotides is not uniform, even under a constant bias. Although the $20 \mathrm{~V}$ bias used in our simulations is strong enough to force DNA through the pore at a high rate (up to 10 nucleotides per ns), a moderate reduction of the bias, for example to $10 \mathrm{~V}$, leads to a dramatic reduction of the DNA translocation rate (by a factor of 40 for $(\mathrm{dA})_{25}$, Fig. S5). Often, the translocation was observed to stall when a new base tried to enter the pore's constriction (Fig. S6). However, due to the high bias used in our simulations, such step-like motion was not always observed. To demonstrate that the translocation velocity of DNA is controlled by the rate at which the nucleotides enter the pore's constriction, the following statistical analysis was carried out. For each system considered, the average densities of the nucleotide's base and phosphate were computed along the pore axis, separately for either translocation direction. First 100-ps fragments of the trajectory immediately following each voltage switch were discarded to eliminate non-stationary periods from the analysis. The linear density of the nucleotides (or of their constituents) obtained thereby characterizes the probability of finding the nucleotides at certain locations in the pore.

Fig. 5 shows the average density of the adenine nucleotides in the pore. Similar plots were obtained for $(\mathrm{dC})_{25},(\mathrm{dG})_{25}$, and (dT) $)_{25}$ (data not shown). The plots reveal equidistant peaks that pinpoint the locations where the nucleotides pause or stall. For each translocation direction, one of the peaks (denoted as $k$ ) is precisely located at the constriction's entrance, which implies that this peak is due to the nucleotide's pausing before entering the pore. The location of the other peaks appears to strongly correlate with the location of peak $k$. Indeed, the average distances between the $n$ th-nearest-neighbor phosphates have standard deviations that vary from $0.5 \AA$ for $n=1$ to $1.5-2.5 \AA$ for $n=5$. For this reason the peaks of the phosphate's density 
are nearly equidistant and have similar shapes. It can be expected that at a smaller bias, the nucleotides will wait much longer before entering the pore, and that the translocation will occur in a stepper-motor-like manner, where rapid advances of well-defined magnitudes (the nucleotide length, $7.5 \AA$ ) are interspersed with relatively long, controllable pauses. During the pause, the location of each nucleotide in the pore is predetermined, which should facilitate the detection of the nucleotide's type. The translocation of individual nucleotides can be detected by monitoring the potential at the capacitor's electrodes ${ }^{33}$ (Fig. S7).

Shown in Fig. 5c,d are the average induced potentials from individual nucleotides located at their most probable positions, as specified by the peaks of their linear density, Fig. 5a,b. The potentials are plotted as a function of the electrode location. For the top electrode $(z=5 \AA)$ in Fig. $5 \mathrm{c}$ and for the bottom electrode $(z=-5 \AA)$ in Fig. $5 \mathrm{~d}$, the potential induced by the nucleotide nearest to the electrode contributes about $50-60 \%$ of the total potential. The two nearest neighbors of that nucleotide contribute another $\sim 40 \%$ to the total potential, while including the next-nearest neighbors brings the overall contribution close to $100 \%$ (see also Fig. S8).

As shown in Fig. S9 in the supporting information, the potentials induced by individual nucleotides of different types are statistically different and could be distinguished in experiment if their individual potentials could be measured. Our calculations, however, suggest that the poten- tial measured by the device will include significant contribution from the neighboring nucleotides, and will require a deconvolution procedure before the sequence of the DNA strand can be determined to single nucleotide resolution. Knowing even a partial sequence of the strand would significantly simplify the deconvolution.

Fig. 6 compares the translocational dynamics of the four DNA homopolymers under the alternating field conditions. For our analysis, it is convenient to quantitatively characterize DNA transport by computing the cumulative number of nucleotides $N$ passing through the pore constriction or, for definiteness, the plane $z=0$. Fig. 6a shows $N(t)$ for the four simulated system. Combining segments from each dependence that correspond to the same polarity of the driving bias, effective constant-bias dependences $\tilde{N}^{+}(t)$ or $\tilde{N}^{-}(t)$ are constructed. The slope of each such plot gives the average translocation velocity. To estimate the slope and the error of estimate, we considered the correlation function, $v^{ \pm}(t, \tau)=\left(\tilde{N}^{ \pm}(t+\tau)-\tilde{N}(t)\right) / \tau$. The average of this function and its standard deviation computed over all data points using $\tau=10 \mathrm{~ns}$ are plotted in Fig. 6b. For the adenine and cytosine homopolymers, we observed faster translocation toward the $3^{\prime}$ end of the strand than toward the $5^{\prime}$ end of the strand, in agreement with simulations and experiments carried out using the $\alpha$-hemolysin channel. ${ }^{31}$ Surprisingly, for the thymine homopolymer we observed equal translocation velocities for both orientations of the DNA strand, while the guanine homopolymer was observed to move faster in the 5' direction. Similar ratios of the translocation velocities were obtained from the simulations of the $(\mathrm{dT})_{70}$ and $(\mathrm{dG})_{70}$ strands moving through the same pore at constant biases $( \pm 20 \mathrm{~V})$. Note that in the case of $\alpha$-hemolysin, MD simulations predict faster translocation toward the 3'-end of the strand for all types of the DNA homopolymers (unpublished).

The observed differences in the translocation velocities are arguably large enough to be employed to determine the sequence of a DNA strand. It is most straightforward to identify the sequence of nucleotides of the same type: an alternating field that can force DNA to move by exactly $n$ nucleotides back and forth will have different frequencies and profiles for the fragments of C, T, G and A nucleotides. Reading a random sequence is more cumbersome but may still be possible because the duration of the pause separating the one-nucleotide displacements of the DNA strand depends mainly on the type of the nucleotide that is about to enter the pore. 
To demonstrate the feasibility of detecting a single nucleotide substitution using an alternating bias, we simulated two systems - $(\mathrm{dC})_{25}$ and $\mathrm{d}\left(\mathrm{C}_{15} \mathrm{AC}_{9}\right)$ - applying a voltage ramp of $16 \mathrm{~V} /$ ns. In the second system, the mutated nucleotide was located just above the constriction, Fig. $6 \mathrm{~d}$,e. Each system was equilibrated for $6 \mathrm{~ns}$. Five states, separated by about $0.5 \mathrm{~ns}$, were extracted from the last $2.0 \mathrm{~ns}$ of each equilibration trajectory to act as initial conditions for the subsequent voltage ramp simulations. Fig. 6c juxtaposes the motion of the two molecules under a voltage ramp. To pass through the pore's constriction, the bases of the molecule have to reorient, Fig. 2a-b. We observed that, on average, the onset of translocation occurred near a bias of $11 \mathrm{~V}$ for the cytosine homopolymer while it occurred near $13 \mathrm{~V}$ for the DNA strand having a single nucleotide substitution. Furthermore, the standard deviations of the position distributions did not overlap in this bias range, as shown in Fig. 6c. Hence, by increasing the bias linearly to the critical value required to pull the cytosine nucleotide into the constriction and then switching it off, we can distinguish the sequences to a reasonable degree of accuracy by determining whether the molecule has advanced by one nucleotide or not. We expect that optimization of parameters such as the geometry of the pore and the rate of change of the bias will allow such sequences to be discriminated to even greater accuracy.

Thus, by performing calibration measurements using known DNA sequences, sequencespecific cycles of the driving potential can be designed such that, when applied to a DNA fragment of a matching sequence, produce repetitive motion of that fragment back-and-forth through the pore's constriction. When applied to a random unknown sequence, such an alternating field cycle will cause "resonance"-DNA oscillation at the expected amplitudeonly when the matching fragment is located in the pore. Applied to a non-matching sequence, the field will cause DNA translocation by an incorrect number of steps, or no translocation at all. A proof-of-principle demonstration of such behavior is shown in Fig. 6e. In that respect, the nanopore device operating under a particular sequence-specific voltage pattern may act similarly to a restriction enzyme that slides along DNA until it encounters the matching (cognate) sequence. The difference is that the nanopore can be tuned to be specific to any sequence, and thus act as a programmable restriction enzyme without the cleavage functionality. We recognize that some sequence-specific signals may be too similar to each other and therefore cause a false resonance. Note, however, that the velocity of the DNA translocation dramatically depends on the strength of the driving electric field, and therefore the dependence of the translocation velocity on the DNA sequence is expected to become stronger upon decreasing the strength of the driving field.

In summary, we have demonstrated that DNA motion through a 1-nm-diameter pore under alternating electric field conditions exhibits hysteresis due to tilting of the DNA nucleotides in the pore constriction. Such hysteresis is sequence specific and may be used to detect the sequence of DNA nucleotides confined in the pore constriction by measuring the potentials induced at the capacitor's electrodes or the change of the DNA mobility in the nanopore. Other possible sequencing strategies may rely on detecting the sequence-specific hysteresis by the change of the nanopore's capacitance, or by the change of the leakage tunneling current between the capacitor's electrodes. A successful implementation of the nanopore sequencing device will likely require calibration using DNA homopolymers and repeat copolymers. Further theoretical and computational research is necessary to optimize the pore geometry, to clarify the influence of the nanopore's surface on the translocation dynamics of DNA, and to determine the influence of the explicit ions, water, and mobile charges in the semiconductor domain on the induced potentials.

\section{Supplementary Material}

Refer to Web version on PubMed Central for supplementary material. 


\section{Acknowledgements}

This work was supported by the grants from the National Institutes of Health (PHS 5 P41 RR05969 \& R01-HG003713), and the Department of Physics at the University of Illinois. The authors gratefully acknowledge supercomputer time at the Pittsburgh Supercomputer Center and the National Center for Supercomputing Applications provided via Large Resources Allocation Committee grant MCA05S028, as well as the supercomputer time at the Turing cluster (UIUC).

\section{References}

1. International Human Genome Sequencing Consortium. Nature 2004;431:931-945. [PubMed: 15496913]

2. The International HapMap consortium. Nature 2005;437:1299-1320. [PubMed: 16255080]

3. Service RF. Science 2006;311:1544-1546. [PubMed: 16543431]

4. Bayley H. Current Opinion Chemical Biology 2006;10:628-637.

5. Deamer D, Branton D. Accounts of Chemical Research 2002;35:817-825. [PubMed: 12379134]

6. Dekker, C. 2007. p. 209-215.

7. Kasianowicz JJ, Brandin E, Branton D, Deamer DW. Proceedings of the National Academy of Sciences, USA 1996;93:13770-13773.

8. Akenson M, Branton D, Kasianowicz JJ, Brandin E, Deamer DW. Biophysical Journal 1999;77:32273233. [PubMed: 10585944]

9. Vercoutere W, Winters-Hilt S, Olsen H, Deamer D, Haussler D, Akeson M. Nature Biotechnology 2001;19:248-252.

10. Ashkenasy N, Sánchez-Quesada J, Bayley H, Ghadiri MR. Angewandte Chemie - International Edition in English 2005;44:1401-1404.

11. Heng JB, Ho C, Kim T, Timp R, Aksimentiev A, Grinkova YV, Sligar S, Schulten K, Timp G. Biophysical Journal 2004;87:2905-2911. [PubMed: 15326034]

12. Mara, A.; Siwy, Z.; Trautmann, C.; Wan, J.; Kamme, F. 2004. 0, 0-0

13. Chen P, Gu J, Brandin E, Kim Y-R, Wang Q, Branton D. Nano Lett 2004;4:2293-2298.

14. Chang H, Kosari F, Andreadakis G, Alam MA, Vasmatzis G, Bashir R. Nano Letters 2004;4:15511556.

15. Fologea D, Uplinger J, Thomas B, McNabb DS, Li J. Nano Letters 2005;5:1734-1737. [PubMed: 16159215]

16. Storm AJ, Chen JH, Zandbergen HW, Dekker C. Physical Review E 2005;71:051903-051913.

17. Zwolak M, Ventra MD. Nano Letters 2005;5:421-424. [PubMed: 15755087]

18. Lagerqvist J, Zwolak M, Ventra MD. Nano Letters 2006;6:779-782. [PubMed: 16608283]

19. Li J, Stein D, McMullan C, Branton D, Aziz MJ, Golovchenko JA. Nature 2001;412:166-169. [PubMed: 11449268]

20. Storm AJ, Chen JH, Ling XS, Zandergen HW, Dekker C. Nature Materials 2003;2:537-540.

21. Heng JB, Aksimentiev A, Ho C, Dimitrov V, Sorsch T, Miner J, Mansfield W, Schulten K, Timp G. Bell Labs Technical Journal 2005;10:5-22. [PubMed: 18815623]

22. Gracheva ME, Xiong A, Leburton J-P, Aksimentiev A, Schulten K, Timp G. Nanotechnology 2006;17:622-633.

23. Dimitrov, V.; Yemenicioglu, S.; Timp, G. Submitted

24. Aksimentiev A, Heng JB, Timp G, Schulten K. Biophysical Journal 2004;87:2086-2097. [PubMed: 15345583]

25. Ho C, Qiao R, Chatterjee A, Timp RJ, Aluru NR, Timp G. Proceedings of the National Academy of Sciences, USA 2005;102:10445-14450.

26. Kim D, Park J, Shin J, Kim P, Lim G, Shoji S. Sensor Actuat. B-Chem 2006;117:488-494.

27. Allen, MP.; Tildesley, DJ. Computer Simulation of Liquids. Oxford University Press; New York: 1987.

28. Phillips JC, Braun R, Wang W, Gumbart J, Tajkhorshid E, Villa E, Chipot C, Skeel RD, Kale L, Schulten K. Journal of Computational Chemistry 2005;26:1781-1802. [PubMed: 16222654] 
29. Cornell WD, Cieplak P, Bayly CI, Gould IR, Merz KM Jr. Ferguson DM, Spellmeyer DC, Fox T, Caldwell JW, Kollman PA. Journal of the American Chemical Society 1995;117:5179-5197.

30. Heng JB, Aksimentiev A, Ho C, Marks P, Grinkova YV, Sligar S, Schulten K, Timp G. Biophysical Journal 2006;90:1098-1106. [PubMed: 16284270]

31. Mathé J, Aksimentiev A, Nelson DR, Schulten K, Meller A. Proceedings of the National Academy of Sciences, USA 2005;102:12377-12382.

32. Wang Y, Rader AJ, Bahar I, Jernigan RL. Journal of Structural Biology 2004;147:302-314. [PubMed: 15450299]

33. Gracheva ME, Aksimentiev A, Leburton J-P. Nanotechnology 2006;17:3160-3165. 


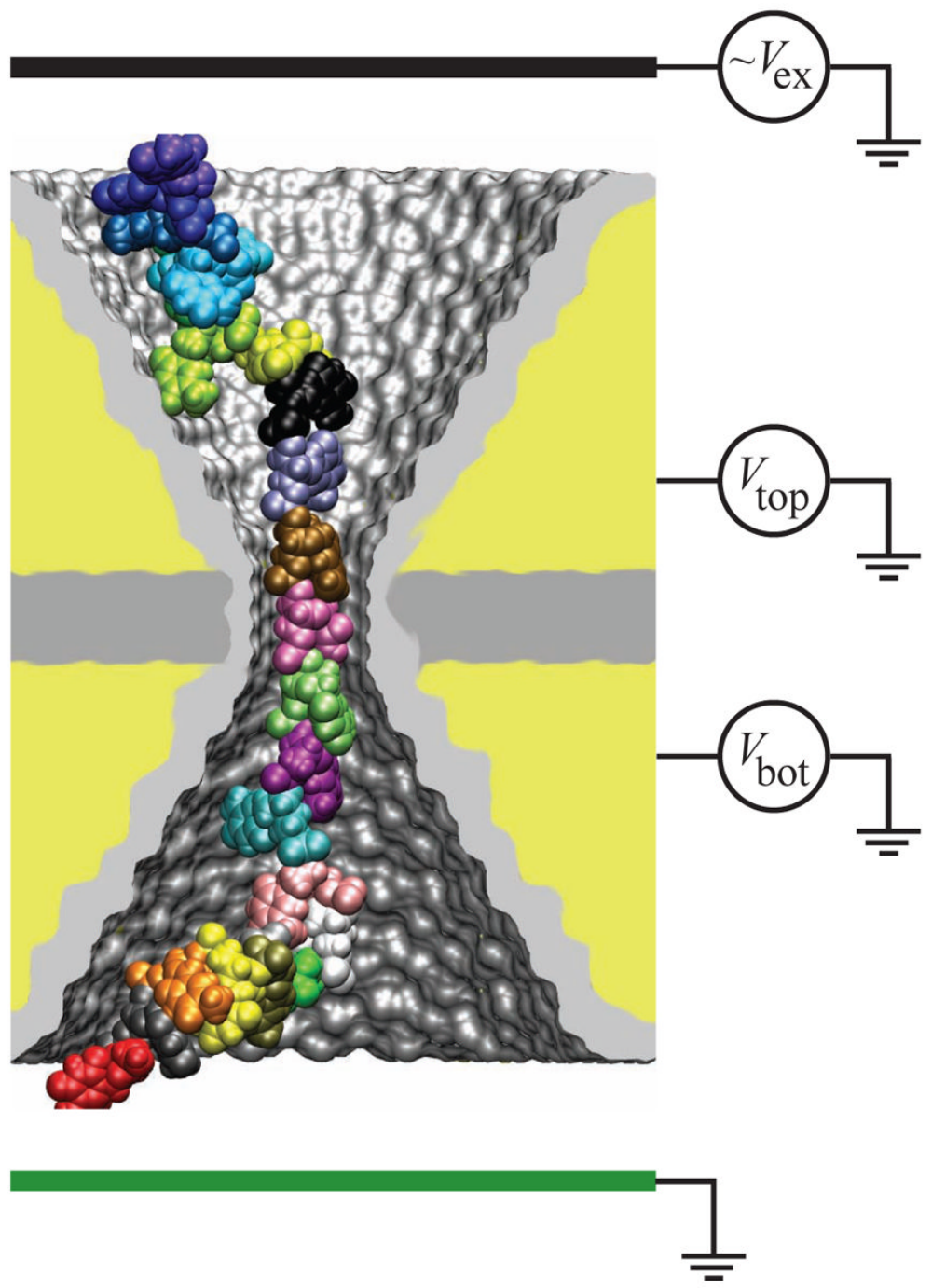

Figure 1.

Schematics of the nanopore device for sequencing DNA. An alternating external bias $V_{\mathrm{ex}}$ drives a single DNA strand back and forth through a nanometer-diameter pore in a synthetic membrane submerged in electrolyte solution. Shown in gray is insulating $\mathrm{SiO}_{2}$, which separates two conducting plates made of highly doped silicon (shown in yellow). $\mathrm{SiO}_{2}$ is also present at the pore's surface. The silicon layers are used as electrodes to measure the electrostatic potentials induced by DNA motion in the pore. 

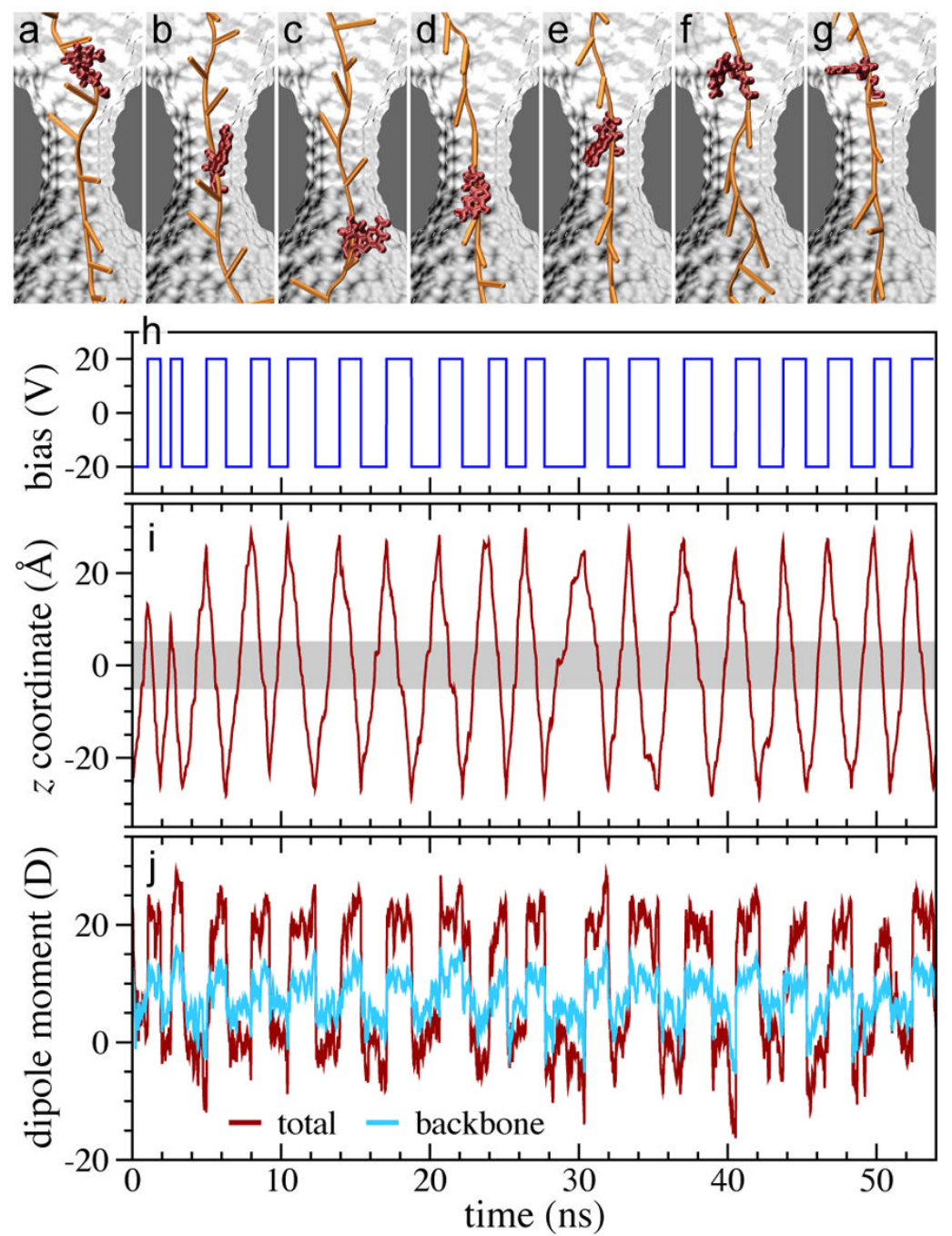

Figure 2.

DNA motion under alternating external electric field. (a-g) Typical conformations of a DNA strand during one translocation cycle. The conformations of one nucleotide are highlighted for clarity. (a-b) DNA moves down (toward its 5' end) with its bases tilted up; (c) the base of the highlighted nucleotide reorients after the voltage's polarity has been reversed; (d-f) DNA moves up with its bases tilted down; $(\mathrm{g})$ the base of the selected nucleotide changes its orientation again. (h) The profile of the driving potential. (i) The position of the center-of-mass of a DNA nucleotide from the middle of the DNA strand. The shaded area indicates the location of the pore's constriction. (j) Vertical ( $z$ ) component of the dipole moment of the entire DNA nucleotide (red) and its backbone (cyan) moving back and forth through the pore constriction. These plots were made for the $(\mathrm{dG})_{25}$ system. 


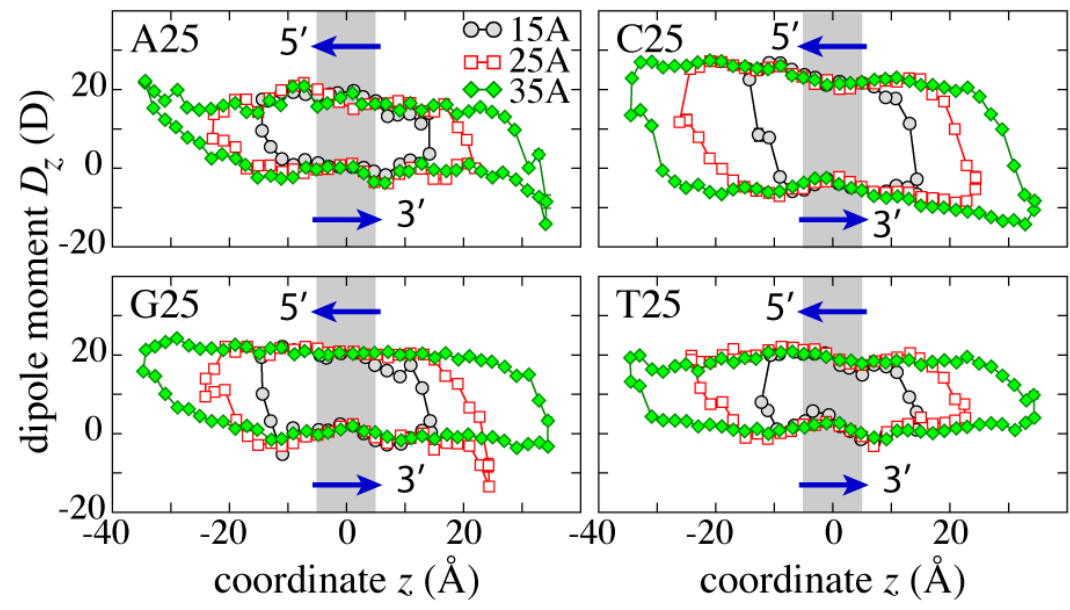

Figure 3.

Hysteresis of nucleotides dipole moment during oscillatory translocation. The average projection of the nucleotide dipole moment onto the pore axis ( $z$ axis) was calculated as a function of the nucleotides center-of-mass position $z$ and DNA translocation direction (shown by arrows). The data were grouped according to the effective amplitude of DNA oscillation $z^{*}$ (see text for details). In each panel, the looped curves show the periodic change in the dipole moment of a single nucleotide during DNA oscillation at an amplitude of $z^{*}=15 \pm 5,25 \pm 5$, and $35 \pm 5 \AA$. The shaded area indicates the location of the pore constriction. 

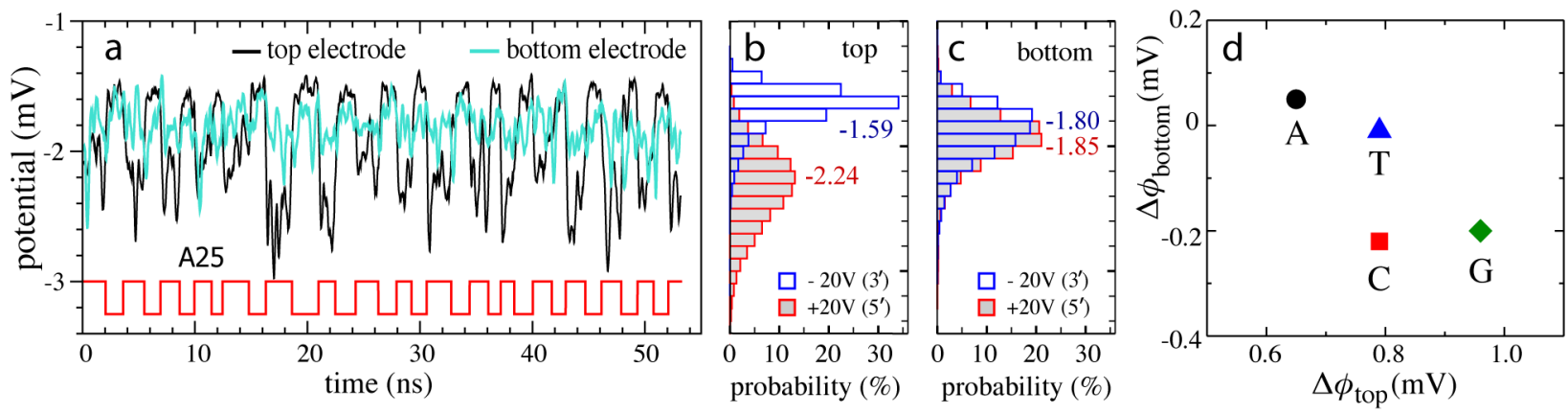

Figure 4.

Electrostatic potentials at the capacitor's electrodes induced by the DNA's charge. (a) Induced potential at the top (black) and the bottom (cyan) electrodes computed from the 54-ns MD trajectory of $(\mathrm{dA})_{25}$. The driving square-wave potential of $\pm 20 \mathrm{~V}$ is shown by the red line (the amplitude is not shown to scale). (b,c) The normalized distribution of the induced potential at the top (b) and the bottom (c) electrodes. In each panel, two distributions are plotted,

distinguished by the polarity of the driving potential. The average values of the induced potentials (in $\mathrm{mV}$ ) are indicated in each panel. Data for $(\mathrm{dC})_{25},(\mathrm{dG})_{25}$, and $(\mathrm{dT})_{25}$ are presented in Fig. S1 in the supporting information. (d) Change of the electrostatic potential at the top and bottom electrodes of the nanopore capacitor upon switching the polarity of the driving bias. From this plot, homopolymers of A, C, G, and T nucleotides can be uniquely identified. In our systems, the $-20 \mathrm{~V}$ bias drives the DNA strand toward its $3^{\prime}$ end. 

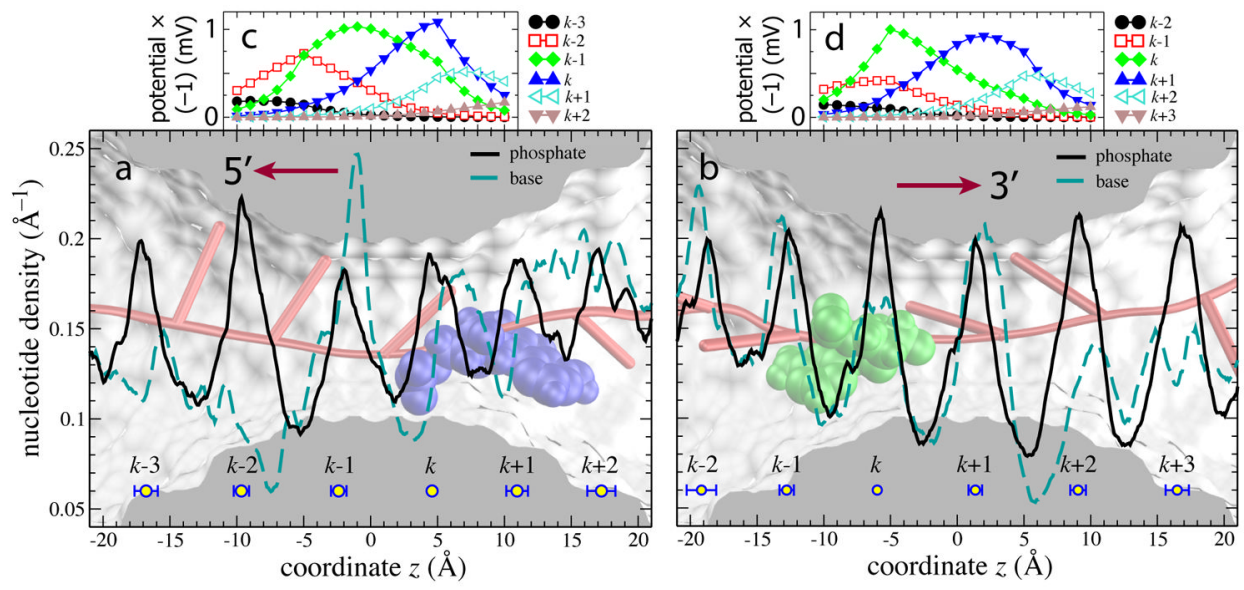

Figure 5.

Linear density of the DNA nucleotides in a 1-nm-diameter nanopore. (a-b) Linear densities of $(\mathrm{dA})_{25}$ 's phosphates (solid line) and bases (dashed line) averaged over the MD trajectory. The densities are computed separately for the fragments of the trajectory that correspond to the same polarity of the transmembrane bias. $(\mathrm{dA})_{25}$ translocates toward its $5^{\prime}$ end in (a) and toward its $3^{\prime}$ end in (b). For each translocation direction, $k$ th phosphate stalls at the pore's entrance. Assuming the most probable position of the $k$ th phosphate, the average coordinates of the neighboring phosphates are shown by circles. The error bars represent the corresponding standard deviations of the average. Typical conformations of the DNA strand are depicted in the background images. (c-d) The electrostatic potentials of the individual nucleotides located at their most probable positions, i.e., at the peaks of the corresponding linear density distributions, numbered accordingly. The potentials are plotted as a function of the electrode location. 

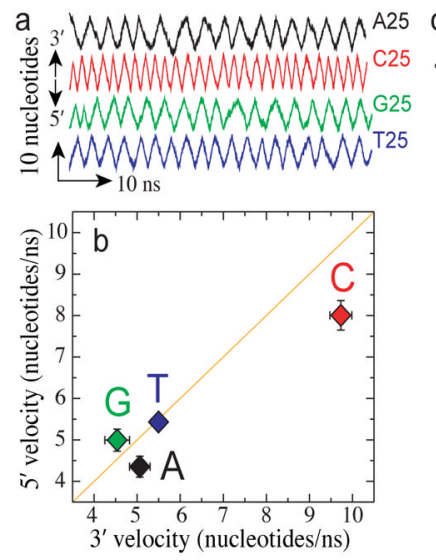
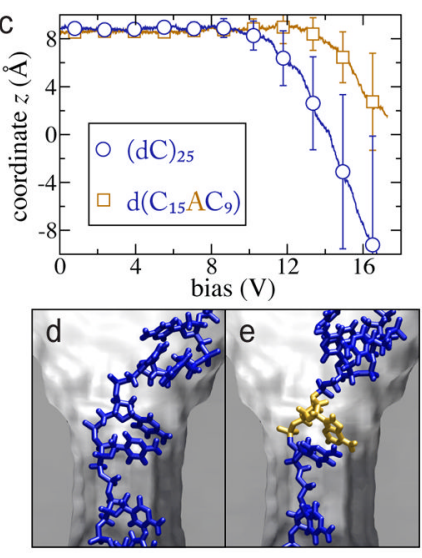

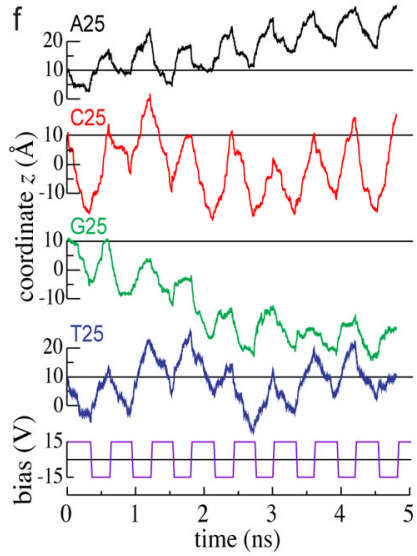

Figure 6.

Sequence dependence of DNA translocation under time-dependent transmembrane biases. (a) The cumulative number of nucleotides passing through the pore constriction versus the simulation time. (b) The average velocity of the $3^{\prime}$ end-bound (abscissa) and $5^{\prime}$ end-bound (ordinate) translocation for each type of the DNA homopolymer. The error bars represent the standard deviations. (c) Effect of single nucleotide substitution on DNA motion under a voltage ramp. The center of mass of the nucleotide initially immediately above the constriction is plotted for $(\mathrm{dC})_{25}$ (blue circles) and $\left.\mathrm{d}_{\left(\mathrm{C}_{15} \mathrm{AC}\right.}\right)$ (gold squares). The trace shown for each system is the average over five MD trajectories while the error bars show the associated standard deviations. The onset of motion occurs at a significantly lower bias for cytosine than for adenine, on average. (d,e) Snapshots of selected initial conditions for the voltage ramp simulations described in panel (c). (f) Motion of DNA homopolymers subject to an asymmetric square pulse bias. The bias produces oscillations of $(\mathrm{dC})_{25}$ about its mean position in the nanopore whilst $(\mathrm{dA})_{25}$ and $(\mathrm{dG})_{25}$ drift in opposite directions. Note that the amplitude and character of oscillations for $(\mathrm{dT})_{25}$ is different than that for $(\mathrm{dC})_{25}$. 